ROCKY MOUNTAIN

JOURNAL OF MATHEMATICS

Volume 9, Number 2, Spring 1979

\title{
ON THE SUPPORT OF THE RADON TRANSFORM
}

\section{STEPHEN ANDREA}

A basic tool in the study of hyperbolic equations is the Radon transform: if $f(x)$ is a function defined in $R^{n}$, then one takes $R f(s, \omega)=\int_{P} f(x) d \sigma$, where $s \in R, \omega \in R^{n}$ has norm one, $P$ is the hyperplane in $R^{n}$ given by $\{x \in R / x \cdot \omega=s\}$, and $d \sigma$ is the element of $(n-1)$-dimensional measure.

The domain of the Radon transform can be taken to be $\mathscr{P}\left(R^{n}\right)$, the space of smooth functions which, together with all derivatives, vanish rapidly at infinity. For this case, it turns out that the Radon transform is in $\mathscr{P}\left(R \times S^{n-1}\right)$.

Now, one sees directly that if $f$ were in $C_{0}{ }^{\infty}\left(R^{n}\right)$ and had support in $|x| \leqq M$, then $R f(s, \omega)$ would vanish for all $|s| \geqq M$, that is, $\int_{P} f(x) d \sigma=0$ for all hyperplanes $P$ in $R^{n}$ whose distance to the origin is at least $M$.

It is an interesting result that the converse statement is true.

THEOREM. Let $f \in \mathscr{P}\left(R^{n}\right)$, and suppose that $\int_{P} f d \sigma=0$ for all hyperplanes $P$ in $R^{n}$ whose distance to the origin is greater than some $M>0$. Then in fact $f \in C_{0}{ }^{\infty}\left(R^{n}\right)$, with support in $|x| \leqq M$.

This fact appears in Ludwig [1] and Lax-Phillips [2], where its proof depends on spherical harmonic expansions and the Paley-Wiener theorem. The present note will give a direct proof.

\section{STEP ONE. If the theorem holds in $R^{n+1}$ then it holds in $R^{n}$.}

To prove this, take $f \in \mathscr{P}\left(R^{n}\right)$. Then pass to $g \in \mathscr{P}\left(R^{n+1}\right)$ where $g(x, t)=f(x) \phi(t)$, with $x \in R^{n}, t \in R$, and $\phi \in C_{0}{ }^{\infty}(R)$. Suppose $\phi$ has support in $-\epsilon \leqq t \leqq \epsilon$.

Suppose $\int_{P_{n-1}} f d \sigma_{n-1}=0$ for all hyperplanes $P_{n-1}$ in $R^{n}$ whose distance to the origin is at least $M \geqq 0$. Then consider $\int_{P_{n}} g d \sigma_{n}$, where $P_{n}$ is a hyperplane in $R^{n+1}$ whose distance to the origin is at least $\sqrt{M^{2}+\epsilon^{2}}$.

If $\operatorname{supp}(g) \cap P_{n}=\phi$ then $\int_{P_{n}} g d \sigma_{n}=0$. If not, then for each $t \in R$ let $P_{n-1}(t) \subset R^{n}$ denote the hyperplane $\left\{x \in R^{n} /(x, t) \in P_{n} \subset R^{n+1}\right\}$. If $|t|<\epsilon$ the geometry of the situation shows that $P_{n-1}(t)$ will have a distance at least $M$ to the origin of $R^{n}$.

Received by the editors on February 28, 1977 and in revised form on May 9, 1977.

Copyright $\odot 1979$ Rocky Mountain Mathematical Consortium 


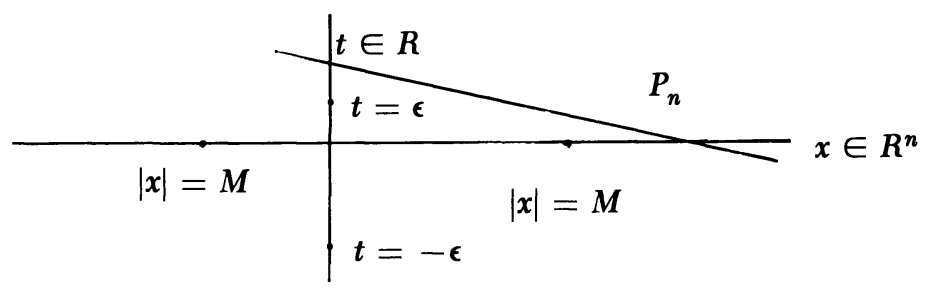

Now $\int_{P_{n}} g d \sigma_{n}=$ (constant) $\times \int_{-\epsilon}^{+\epsilon} \phi(t) \int_{P_{n-1}(t)} f(x) d \sigma_{n-1} d t$. Since the integrand vanishes by the hypothesis on $f$, we see that $\int_{P_{n}} g d \sigma_{n}=0$ for all hyperplanes in $R^{n+1}$ sufficiently distanct from the origin.

Thus the theorem can be applied in $R^{n+1}$, showing that $f(x) \phi(t)$ has support in $|x|^{2}+t^{2} \leqq M^{2}+\epsilon^{2}$. Letting $\epsilon$ pass to zero, one gets the result for $f \in \mathscr{P}\left(R^{n}\right)$.

From now one can assume that $n \geqq 3$, and is odd.

STEP Two. The theorem holds when $f(x)$ is radial.

For, taking $f(x)=\phi(|x|)$ where $\phi: R \rightarrow R$, one can use the particular hyperplane

$$
P(s)=\left\{x \in R^{n} / x_{1}=s\right\}, \text { for } s>0 .
$$

Then one gets

$$
\begin{aligned}
\int_{P(s)} f(x) d \sigma & =\text { const. } \quad \int_{0}^{\infty} \phi\left(\sqrt{s^{2}+r^{2}}\right) r^{n-2} d r \\
& =\text { const. } F(s),
\end{aligned}
$$

where $F(s)=\int_{s}^{\infty} \phi(t)\left(t^{2}-s^{2}\right)^{(n-3) / 2} t d t$, via the change of variables $s^{2}+r^{2}=t^{2}$. Moreover, $F(s)=0$ in $s>M$.

Now the exponent $(n-3) / 2=m$ is a nonnegative integer; for the case $n=3$ and $m=0$ we have $F^{\prime}(s)=-s \phi(s)$, proving that $\phi(s)=0$ in $s>M$ as desired.

For $m \geqq 1$ we have

$$
-\frac{1}{2 m s} \frac{d}{d s} F(s)=\int_{s}^{\infty} \phi(t)\left(t^{2}-s^{2}\right)^{m-1} t d t .
$$

Thus $\phi(s)$ can always be obtained from $F(s)$ by successively dividing by $s$ and differentiating. Hence $F(s)=0$ in $s>M$ implies that $\phi(s)=0$ in $s>M$.

STEP THREE. Given $f \in \mathscr{P}\left(R^{n}\right)$ with $\int_{P} f d \sigma=0$ for all hyperplanes $P \subset R^{n}$ whose distance to the origin is at least $M$. Then $\int_{|x|=r} f(x) d \sigma=0$ for all $r \geqq M$. 
To prove this, consider the functions $f(V x)$ where $V$ ranges over the orthogonal linear transformations of $R^{n}$ to itself.

Take $g(x)=\int_{V \in O(n)} f(V x)$, with integration by Haar measure in the compact group $0(n)$. Evidently $g(x)=g\left(V_{0} x\right)$, showing that $g$ is constant over the spheres $|x|=r$. To identify $g$, note that $\int_{|x|=r} f(V x) d \sigma=$ $\int_{|x|=r} f(x) d \sigma$ for all $V \in 0(n)$; then, changing the order of integration in

$$
\int_{|x|=r} d \sigma \int_{V \in 0(n)} f(V x),
$$

one sees that $\int_{|x|=r} g(x) d \sigma$ is equal to $C_{0} \int_{|x|=r} f(x) d \sigma$, where $C_{0}$ is the total measure of $0(n)$. Since $g$ is radial, we have that $g(x)$ is a nonzero multiple of the integral of $f$ over the sphere of radius $|x|$.

But the transformations $V$ preserve hyperplanes and their distances. Thus each function $x \mapsto f(V x)$ satisfies the hypothesis, as does their average $g(x)$. Now, $g(x) \in \mathcal{P}$ : since $g$ is radial we must have $g \equiv 0$ for $|x|>M$, according to Step Two. This gives the result.

STEP Four. Given $f \in \mathscr{P}\left(R^{n}\right)$, with $\int_{P} f d \sigma=0$ for hyperplanes $P \subset R^{n}$ whose distance to the origin is greater than $M>0$. Then $f(x)=0$ for $|x|>M$.

Take $\epsilon>0$. Then, for $a \in R^{n}$ with $|a|<\epsilon$, the displaced function $f(x+a)$ will continue to satisfy the hypothesis, but with the slightly larger constant $M+\epsilon$. From the preceding step we have

$$
\int_{|x-a|=r} f(x) d \sigma=0,
$$

valid when $|a|<\epsilon$ and $r>M+\epsilon$.

Put $a=a_{0}+t E_{j}$ where $E_{j}$ is the unit coordinate vector in the $j$ th direction. We differentiate the equation with respect to $t$ at $t=0$, recalling that the finite difference quotients of $f$ converge uniformly to $\partial f / \partial x_{j}$. The result is that

$$
\int_{|x-a|=r} \frac{\partial f}{\partial x_{1}}(x) d \sigma=0
$$

in the same range of $r$ and $a$.

Now take the vector field $V=f(x) E_{j}$, whose divergence is $\partial f / \partial x_{j}$. From the preceding formula we have

$$
\int_{r<|x-a|<R} \operatorname{div} V d R^{n}=0,
$$


valid for $|a|<\epsilon, M+\epsilon<r<R$. Gauss' theorem gives

$$
\int_{|x-a|=r} V \cdot N d \sigma=\int_{|x-a|=R} V \cdot N d \sigma .
$$

The second integral tends to zero with large $R$ because $f$ and its derivatives decrease rapidly and uniformly. Hence

$$
\int_{|x-a|=r} V . N d \sigma=\int_{|x-a|=r} f(x) \frac{x_{j}-a_{j}}{|x-a|} d \sigma=0,
$$

when $r>M+\epsilon$.

The foregoing has established that if $f$ satisfies $\int_{|x-a|=r} f(x) d \sigma=0$ for all $r>M+\epsilon$ and $|a|<\epsilon$, then $x_{j} f(x)$ will have the same property. By repeated application, one sees that every polynomial multiple $P(x) f(x)$ will have a zero integral over the relevant spheres.

But then $f(x) \equiv 0$ in $|x|>M+\epsilon$ by Weierstrass approximation.

This completes the proof of the theorem.

\section{REFERENCES}

1. Donald Ludwig, The Radon Transform on Euclidean Space, Comm. Pure Appl. Math., XIX, (1966), 49-81.

2. P. D. Lax and R. S. Phillips, The Paley-Wiener Theorem for the Radon Transform, Comm. Pure Appl. Math. XXIII (1970), 409-424.

Departmento de Matemáticas, la Universidad Simon Bolivar, Apartado Postal \# 80659 Caracas, Venezuela 